\title{
Migración rural, remesas y su relación con la diversificación sustentable y los patrones de consumo. Un estudio de caso en la zona costera de Oaxaca'
}

\author{
Rural migration, remittances and their bond with the sustainable \\ diversification and the consumption patterns. \\ A case study in the coast of Oaxaca
}

\author{
Ana María Aragonés* \\ Sophie Ávila* \\ Uberto Salgado*
}

\begin{abstract}
RESUMEN: Gran parte de la discusión sobre el impacto que tienen las remesas en las comunidades de origen de los migrantes se centra en el debate de la migración y el desarrollo, sin considerar los efectos que dicha relación provoca sobre el medio ambiente. En ese sentido, este trabajo analiza el papel de las remesas en el desarrollo sustentable a través de la diversificación sustentable y los patrones de consumo entre los hogares receptores y no receptores de la costa oaxaqueña, zona que destaca por una importante actividad ecoturística. Los resultados muestran que las remesas tienen un impacto muy limitado en torno a la diversificación sustentable debido a que el principal uso del recurso es el gasto corriente del hogar; sin embargo, es posible observar un cambio en el patrón de consumo en los hogares receptores que sugiere un efecto positivo sobre el medio ambiente al permitirles reducir el uso de los recursos naturales locales.

PALABRAS CLAVE: desarrollo y diversificación sustentable, consumo conspicuo, patrones de consumo, enfoques funcionalista y estructuralista, costa de Oaxaca.
\end{abstract}

\begin{abstract}
Much of the discussion about remittances impact have on the communities of origin focuses on the debate between migration and development, without considering the effects that this relationship causes on the environment. In that sense, this paper analyzes the purpose of remittances in sustainable development through sustainable diversification and consumption patterns between recipient households and non-recipient households of the coast of Oaxaca, an area that highligths for an important ecoturism activity. The results demonstrate that remittances have limited impact in the sustainable diversification due to the main use of the resource is the current expenditure of the household; however, there is a change in recipient households consumption pattern. This suggest a positive consequence on the environment with the reduction of use of local natural resources.

KEY WORDS: sustainable development and diversification, conspicuos consumption, consumption patterns, functionalist and structuralist approaches, coast of Oaxaca.

${ }_{1}^{1}$ Agradecemos el apoyo de los proyectos Conacyt CB-2010-01-152298 y DGAPA-UNAM PAPIIT IN300716, los cuales permitieron desarrollar el presente estudio. Agradecemos también al Instituto de Investigaciones Económicas por las facilidades prestadas para la buena realización del proyecto y a los becarios Dorian Ivvone Martínez de Lara, Luis Fernando González Martínez, Ulises Sánchez Guerrero y José Rafael Valencia González, cuya colaboración fue destacada.
\end{abstract}





\section{Introducción}

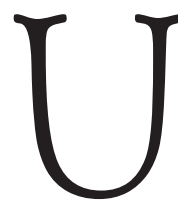

no de los temas centrales desde hace algunos años entre los investigadores de la migración es el análisis y la explicación del vínculo que existe entre la migración y el medio ambiente, gran parte de la literatura se ha centrado en estudiar la manera en que las condiciones ambientales incentivan a la migración (Bilsborrow, 2015); sin embargo, recientemente se ha estudiado cómo la migración puede afectar al medio ambiente. Una de esas líneas de investigación analiza la forma en que la migración rural y las remesas podrían afectar al medio ambiente en sus comunidades de origen (Hecht et al., 2012). Este es un estudio que al adquirir complejidad con el paso del tiempo ha generado diversas perspectivas teóricas para su comprensión. Un elemento importante a destacar es que todos los conceptos mencionados - migración, remesas, medio ambiente - tienen sus propias determinaciones y se explican a partir de visiones teórico-metodológicas muy diversas, por momentos contradictorias entre sí, así como sus conclusiones. No obstante, los tres conceptos tienen una preocupación económico-social que los abarca y es el término de su relación con el fenómeno del desarrollo. Es decir, cuando se estudia la dupla migración-remesas uno de los ejes de discusión gira en torno a debatir si éstas favorecen o no el desarrollo de sus comunidades (Binford, 2002). Y en cuanto a la cuestión del medio ambiente, las principales corrientes del pensamiento ambientalista también se centran en el concepto de «desarrollo sustentable» (Gallegos, s.f.). Al respecto, las tres variables son fenómenos económico-sociales y parece necesario acudir a estudios multidisciplinarios para su profundización y evitar la simple yuxtaposición. Por lo tanto, es conveniente estudiar el desarrollo con esos tres grandes temas.

En este artículo abordamos, en primer término, algunas de las perspectivas que estudian la relación migración-remesas y el papel que desempeñan con relación al desarrollo. En segundo término, presentamos ciertas visiones creadas en el marco de los estudios ambientales para analizar las consecuencias

\footnotetext{
* Profesores investigadores del Instituto de Investigaciones Económicas, Universidad Nacional Autónoma de México.
} 
que el fenómeno migratorio y las remesas pueden tener sobre el medio ambiente de las comunidades receptoras y si favorecen su desarrollo sustentable. El estudio se llevó a cabo en cuatro comunidades de la costa de Oaxaca ubicadas en dos municipios costeros, Santa María Tonameca y Santa María Colotepec, donde el sector turismo (con énfasis en el ecoturismo) y los servicios son fuentes importantes de empleo entre sus habitantes. Por tal motivo, es de gran interés estudiar la relación migración, remesas y desarrollo sustentable en dicha región.

\section{Breve revisión de la relación migración, remesas y desarrollo}

La migración es un fenómeno histórico, y por lo tanto cambiante, de ahí que resulte central analizar sus características en el marco de la globalización, periodo que se inauguró con la crisis de los años 1980 y que supuso la conformación de un nuevo patrón migratorio derivado del nuevo régimen de acumulación (Aragonés y Salgado, 2011a). Una primera característica es el intenso movimiento de personas que se desplazan a lo largo y ancho del planeta, flujos formados en esencia por trabajadores migrantes internacionales. Se trata de personas que se han visto forzadas a buscar nuevos horizontes de vida en la medida en que se ha aplicado el modelo económico neoliberal en la globalización. Proyecto de alcance mundial que ha perjudicado sobre todo a los países subdesarrollados al propiciar una situación de enorme vulnerabilidad para los trabajadores, en especial para los trabajadores rurales, lo que explica, en parte, los enormes flujos migratorios internacionales. Un rasgo distintivo de estos flujos ha sido el compromiso de los migrantes con sus familias y comunidades reflejado en cuantiosos envíos de dinero. Las crecientes remesas han generado flujos financieros de enorme significación cuyo incremento es directamente proporcional al del incremento de trabajadores migrantes internacionales. Esa dinámica mundial ha llamado la atención de investigadores, académicos, ONG y organismos internacionales, interesados en comprender las consecuencias de las remesas en sus lugares de origen. Habría que preguntarse si estos ingresos 
crean nuevas condiciones económicas para las familias y las comunidades de los migrantes que les permitan iniciar procesos de desarrollo sustentable.

Son dos las visiones esenciales en el estudio de la relación migración-remesas: la funcionalista y la histórico-estructuralista. La primera supone que las remesas tienen la posibilidad de reducir la pobreza y la desigualdad del ingreso y ayudan a estabilizar económicamente a las familias. Si bien se destinan a gastos básicos como alimentación, educación, salud, vivienda, entrañan una mejoría de las condiciones de vida con un efecto multiplicador para la economía de las comunidades. Los autores que la defienden plantean que hay elementos para sostener que las remesas ostentan la capacidad para generar desarrollo (Jones, 1995; Massey y Parrado, 1998; Adelman y Taylor, 1990).

En contraste, para la perspectiva histórico-estructuralista las remesas crean una serie de distorsiones estructurales que se reflejan en la exacerbación del conflicto social, así como en diferencias económicas e inflación. Todo ello fomenta un círculo vicioso que distorsiona a la economía local y deteriora sus estructuras sociales. En la medida en que sólo gracias a las remesas las familias pueden mantener ciertas condiciones de vida porque no hay alternativas para acceder a empleos decentes, ${ }^{1}$ la emigración se convierte en una imperiosa necesidad. Es decir, las remesas familiares han sido insuficientes para iniciar procesos de desarrollo de la comunidad, pues se dedican a satisfacer el consumo básico y los escasísimos proyectos productivos que han intentado tienen resultados muy pobres, ya que los beneficios son casi nulos, lo que ocasiona que su duración sea limitada (Stefoni, 2011).

Aun cuando las dos visiones plantean divergencias referentes a la potencialidad de las remesas para propiciar desarrollo, no hay duda de que dadas las condiciones de pobreza y de falta de alternativas económicas que enfrentan las

\footnotetext{
${ }^{1}$ De acuerdo con la Organización Internacional del Trabajo, el concepto de trabajo decente abarca cuatro elementos: el empleo, la protección social, los derechos de los trabajadores y el diálogo social; el concepto incluye la existencia de empleos suficientes (posibilidades de trabajar), la remuneración, la seguridad en el trabajo, las condiciones laborales salubres, la seguridad social, la seguridad de ingresos, los derechos fundamentales del trabajo (libertad de sindicalización y erradicación de la discriminación laboral, del trabajo forzoso y del trabajo infantil), el derecho a exponer sus opiniones, defender sus intereses, entablar negociaciones con los empleadores y con las autoridades sobre los asuntos relacionados con la actividad laboral (Ghai, 2003).
} 
comunidades expulsoras, ambas propuestas están de acuerdo en el hecho de que recibir un ingreso extra permite a esas poblaciones permanecer en sus comunidades y enfrentar sus carencias con cierto alivio. Esto contituye un beneficio para las familias en esas comunidades, pero en lo que difieren tales visiones es con los proyectos productivos y su capacidad para transformar las condiciones de la comunidad. Un dato que nos permite sostener la incapacidad de las remesas como transformadoras de la realidad es que la intensidad migratoria se ha mantenido prácticamente sin cambios en las comunidades expulsoras; lo mismo sucede con los grados de marginación (Aragonés y Salgado, 2008: 45). En consecuencia, si quieren mitigar en alguna medida las condiciones de pobreza de sus familias, los flujos migratorios deben continuar.

Nuestra posición es que las remesas no favorecen el desarrollo, como plantea la visión histórico-estructuralista. El efecto directo de las remesas es la de reproducir un reservorio de fuerza de trabajo lista para emigrar pues las familias dependen de esos ingresos a fin de enfrentar las condiciones de pobreza y desempleo (Aragonés, 2009; Aragonés y Salgado, 2011b). En investigaciones como la de Aragonés et al. (2008) se advierte que cuando las remesas permiten el consumo de ciertos bienes que no se producen en la región favorecen a comunidades lejanas, o bien, si son productos importados, repercuten de modo negativo porque se incrementa el déficit comercial. Sostenemos que el desarrollo debe ser generado a partir de políticas económicas deliberadas instrumentadas por el Estado, agenda que ha sido olvidada en los países subdesarrollados desde la adopción del modelo neoliberal. Desarrollo implica «a todos los aspectos de la sociedad, debe ser sostenible, equitativo, democrático y sus efectos positivos deben alcanzar a toda la población, sobre todo con relación a salud, educación, infraestructura y empleos formales y de calidad» (Stiglitz, 2006: 54). 


\section{Migración rural, remesas y el uso de los recursos naturales}

Las consecuencias ambientales de la migración y de las remesas son complejas y las visiones que las explican son diversas, en particular cuando se trata de decidir su papel vinculado con el desarrollo sustentable, concepto que también presenta sus dificultades. Desde la década de 1960 se relacionó el deterioro ambiental con el desarrollo en el sentido de que «el desarrollo no tomaba en cuenta el equilibrio con el medio ambiente ni lograba el equilibrio al interior de la sociedad humana» (Foladori y Tommasino, 2000). En la medida en que el trabajo es la esencia de la naturaleza humana, a la vez que transforma la naturaleza modifica asimismo a la sociedad (Foladori, 1999). Por lo tanto, hubo desde entonces una mayor preocupación acerca del daño que este tipo de trabajo podía causar al medio ambiente (Simmons, 1993; Foster, 1994). En tanto que las transformaciones fueron cada vez más profundas a lo largo del siglo XX y se fueron adoptando al planeta en su conjunto, los problemas también fueron ya planetarios, como el calentamiento global, la disminución de la capa de ozono o la pérdida de la biodiversidad (Foladori y Tommasino, 2000).

En el informe de la Comisión Mundial para el Medio Ambiente y Desarrollo (por sus siglas en inglés WCED, 1987) se define el término desarrollo sustentable como «aquel que corresponde a las necesidades del presente de forma igualitaria pero sin comprometer las posibilidades de sobrevivencia y prosperidad de las generaciones futuras» y se establece que «la pobreza, la igualdad y la degradación ambiental no pueden ser analizados de manera aislada». El documento «coloca a la pobreza como una de las causas (y consecuencias) de los problemas ambientales» (Foladori y Tommasino, 2000). De esa manera, el desarrollo sustentable se refiere a tres dimensiones: social, económica y ambiental. En 2015, derivado de los objetivos del milenio, las Naciones Unidas establecieron 17 objetivos para el desarrollo sostenible en los próximos 15 años, en los que se observa una relación estrecha entre aspectos ambientales, de salud, económicos y sociales (ONU, 2015). A una escala local, como son los hogares rurales, la manera de conjuntar objetivos socioeconómicos y ambientales es a través de proyectos productivos sustentables, es decir de una diversificación 
sustentable. La diversificación en los hogares rurales es una tendencia mundial y México no es la excepción, con 49.2 por ciento de los ingresos de los hogares provenientes de actividades no agropecuarias (Cerón y Yúnez-Naude, 2015). Aunque no existe una medición nacional de la importancia de los ingresos provenientes de actividades sustentables, se conocen muchos casos de éxito (Carabias et al., 2010).

Por ello, parece necesario profundizar en el concepto de desarrollo sustentable como posibilidad para superar el fenómeno social de la pobreza, de lo contrario el desarrollo no será tal, sino una simple quimera que sólo podrá amortiguar ciertas consecuencias de la degradación ambiental. Teniendo eso en cuenta, el papel que desempeñen las remesas en torno al desarrollo sustentable dependerá del uso que se le dé a dicho recurso. Como advierten Sherbinin et al. (2008) dicho recurso puede ser invertido en emprendimientos productivos amigables con el medio ambiente o en proyectos de conservación de los recursos naturales, como el ecoturismo. Prácticas que contribuyen con la generación de empleos e ingresos que propician un mayor nivel de bienestar social, pero de forma sustentable.

Recientemente se ha desarrollado una línea de investigación, la cual sugiere que si bien los impactos de las remesas sobre el desarrollo económico son limitados, puesto que gran parte de los recursos se destina al consumo diario, este consumo podría impactar a los recursos naturales en forma indirecta. A tal efecto se le ha denominado "consumo conspicuo» (Davis y Lopez-Carr, 2010). Uno de los argumentos es que los migrantes internacionales están expuestos a una diversidad de normas, actitudes, deseos y conductas, y cuando retornan a sus lugares de origen pueden adoptar nuevas ideas y aspiraciones. Las nuevas conductas son las que dan forma al llamado "consumo conspicuo» (bienes como automóviles, ropa, electrónicos, etcétera) (Davis y Lopez-Carr, 2010). En la medida en que estas comunidades rurales se encuentran muy cerca de las áreas de alta biodiversidad, al «cambiar los estilos de vida, de salud, y el consumo de los hogares» las nuevas condiciones representan un potencial para el cambio ambiental (Qin y Flint, 2012). Es decir, si se acepta que las remesas cambian los patrones de consumo de sus familias en las comunidades receptoras, ese consumo puede afectar a las actividades agrícolas en las tierras locales, 
así como en la construcción de nuevas casas, cambios en el uso de energía y adopción de tecnologías (teléfonos, computadoras, etcétera), lo cual tendría consecuencias ambientales (Zárate-Hoyos, 2004). Lo anterior resulta relevante considerando que el uso de recursos naturales es importante para la sobrevivencia de los hogares, en particular la leña (Fierros y Avila-Foucat, en revisión; Lopez-Feldman, 2014). Es cierto que también este consumo comprende una forma de exhibir cierto estatus social del individuo, por lo que puede ser percibido como un factor de desigualdad social; además, como indica Lipton (1980), el efecto del consumo de bienes importados no sólo eleva el costo de la vida, sino que reduce la fuerza de la productividad para incrementar el bienestar social. Aunque se argumenta que las remesas pueden ser benéficas cuando son sustituidas por bienes locales. Es interesante el ejemplo de los hogares receptores de remesas en Michoacán, las que se emplean para comprar gas propano importado como combustible, reduciendo así el consumo de los recursos forestales cercanos a la comunidad como fuente primaria de energía, lo que ha permitido llevar a cabo una conservación forestal en la región (Klooster, 2003).

Por lo expuesto con anterioridad es claro que se requieren más estudios para seguir profundizando acerca de los diferentes cambios que permite el consumo atribuible a las remesas, en concreto cuando lo que se pretende es asociarlo con el medio ambiente, pues el vínculo es altamente complejo. En el presente trabajo desarrollamos un estudio de caso sobre la relación que existe entre las remesas y el medio ambiente en cuatro comunidades de la costa oaxaqueña. Estudiamos los efectos directos que pueden generar las remesas en el desarrollo económico y el desarrollo económico sustentable al analizar su posible contribución en la construcción de emprendimientos productivos sustentables, como el ecoturismo, actividad de gran trascendencia en la región; después analizamos si las remesas han provocado un cambio en el patrón de consumo en los hogares receptores y las posibles repercusiones sobre el uso de los recursos naturales en la región. 


\section{Contexto de la migración en Oaxaca}

El estado de Oaxaca abarca una superficie de 95364 kilómetros cuadrados, que en términos porcentuales representa 4.8 por ciento de la superficie total del país de acuerdo con cifras de INEGI. De los 570 municipios que lo integran, 50 pertenecen a la Región Costa, que a su vez se divide en tres distritos que comprenden a Jamiltepec, Juquila y Pochutla; en esta última se localizan los municipios de Santa María Colotepec y Santa María Tonameca, en los que se llevó a cabo el trabajo de campo (véase mapa 1).

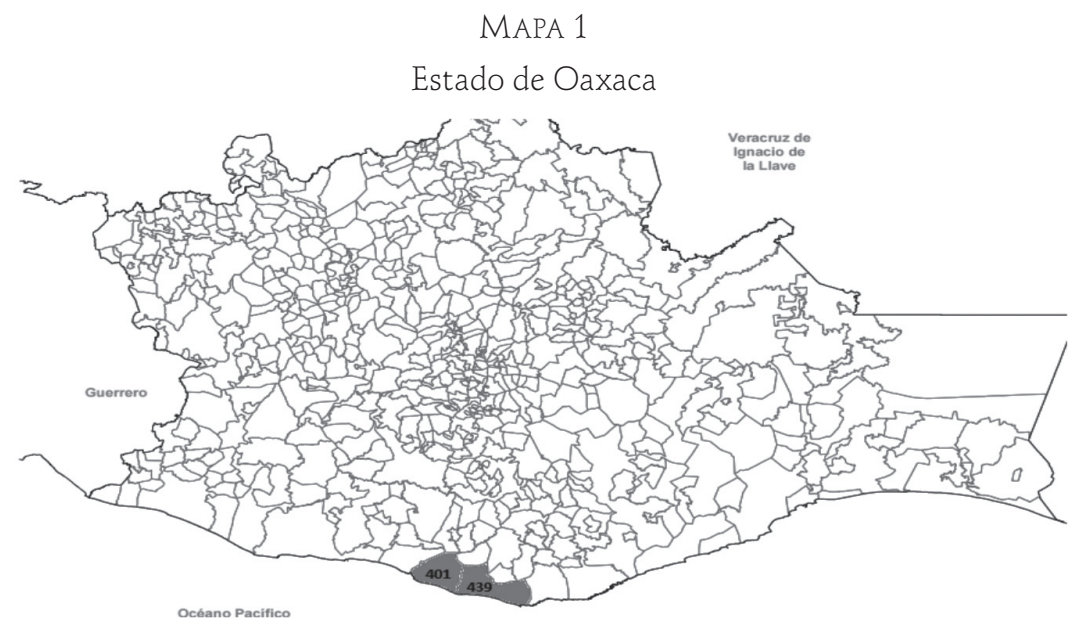

Fuente: imagen tomada del INEGI (2016).

La migración de oaxaqueños hacia Estados Unidos es un fenómeno reciente que se ha presentado en las entidades del sur de la república mexicana, tal como indica Binford (2002: 149) «la migración se ha extendido como un fuego incontrolable» desde 1990 en dicha región. Es relevante destacar que el Índice Absoluto de Intensidad Migratoria (IAIM) de mexicanos hacia Estados Unidos, desarrollado por Conapo, expone que Oaxaca es una de las entidades con mayores tasas de crecimiento entre 2000 y 2010 al ocupar el quinto lugar a nivel nacional, pasando de un grado de intensidad migratoria medio a uno alto para 2010 (véase cuadro 1). 


\section{CUADRO 1}

Índice de Intensidad Migratoria, cambio relativo y cambio absoluto, primeras cinco entidades que experimentaron un incremento entre 2000 y 2010

\begin{tabular}{ccccc}
\hline $\begin{array}{c}\text { Clave de } \\
\text { la entidad } \\
\text { federativa }\end{array}$ & Estado & $\begin{array}{c}\text { Índice absoluto } \\
\text { de intensidad } \\
\text { migratoria 2000 }\end{array}$ & $\begin{array}{c}\text { Índice absoluto } \\
\text { de intensidad } \\
\text { migratoria 2010 }\end{array}$ & Cambio relativo \\
\hline 7 & Chiapas & 0.44 & 0.91 & 106.82 \\
27 & Tabasco & 0.35 & 0.53 & 51.43 \\
29 & Tlaxcala & 1.44 & 2.02 & 40.28 \\
23 & Quintana Roo & 0.51 & 0.70 & 37.25 \\
20 & Oaxaca & 2.56 & 3.23 & 26.17 \\
4 & Campeche & 0.53 & 0.64 & 20.75 \\
26 & Sonora & 1.50 & 1.78 & 18.67 \\
\hline
\end{tabular}

Fuente: datos tomados de Conapo (2014).

Respecto al cambio en la recepción de remesas en México entre 2000 y 2010, siete entidades federativas registraron un incremento; resaltan Oaxaca, Puebla y Baja California con las mayores tasas (Conapo, 2014). Cabe mencionar que en el 2010 las remesas representaron 8.4 por ciento del producto interno bruto estatal oaxaqueño (CEIEG, 2014).

Como se mencionó con anterioridad el fenómeno migratorio en Oaxaca es nuevo y la mayor proporción de estos desplazamientos surgen en la región mixteca. A pesar de que la migración en la región costa es muy inferior (Alvarado, 2008), esta última ha comenzado a mostrar una creciente participación debido a que el índice de intensidad migratoria en Santa María Colotepec pasa de muy bajo a bajo entre 2000 y 2010, mientras que en Santa María Tonameca, pasa de bajo a medio en el mismo periodo.

\section{Características socioeconómicas de Oaxaca y su costa}

En el cuadro 2 se observa que en Oaxaca y en los dos municipios costeros poco más de una tercera parte de su población total se constituye como población económicamente activa y que casi toda se encuentra ocupada. Debido a que 
dichas regiones presentan tasas de desocupación muy bajas, es necesario tomar con precaución dicho dato porque se considera como ocupada a una persona que realice cualquier actividad remunerada, sea en el sector formal o informal de la economía. En consecuencia, es viable considerar que existe una gran participación del trabajo informal que se refleja en el deterioro de su mercado laboral, que se evidencia en distintos indicadores: la población que percibe hasta dos salarios mínimos y la población no derechohabiente con una alta participación a nivel estatal y en ambos municipios.

Aunado a lo anterior, la entidad tiene una tasa de crecimiento económico muy baja, pues en el 2014 fue de 1.96 por ciento, cuando el promedio nacional fue de 2.16 por ciento, en contraste con el primer lugar que ocupa el estado de Nuevo León con 3.86 por ciento. La participación por actividad económica es principalmente la vinculada con el sector terciario con 61 por ciento, la secundaria es de 34 por ciento y la actividad primaria sólo de 6 por ciento (INEGI, 2016). Este débil crecimiento económico, junto con la falta de oportunidades laborales, permite comprender porqué esta población se enfrenta a severos niveles de marginación y pobreza; al respecto, Coneval reporta que Oaxaca es una de las entidades con mayor rezago social y carente de bienestar económico. Para el año 2010 la entidad ocupaba la tercera posición con mayor número de pobres, ya que cerca de 67.4 por ciento de su población total vive bajo esa condición y alrededor de 29.8 por ciento son pobres extremos; a estos datos es necesario añadir que en ambos municipios se observan elevados niveles de pobreza y pobreza extrema (cuadro 2). 


\section{CUADRO 2}

Características del mercado laboral e indicadores de pobreza para Oaxaca y municipios seleccionados, 2010

\begin{tabular}{|c|c|c|}
\hline Estado & 2010 & \\
\hline Oaxaca & Valor absoluto & $\%$ \\
\hline Población total & $3,801,962$ & - \\
\hline PEA & $1,343,189$ & 100.00 \\
\hline Desocupados & 44,873 & 3.34 \\
\hline Ocupados & $1,298,316$ & 100.00 \\
\hline PEA ocupada con ingresos de hasta dos salarios mínimos & 750,037 & 57.77 \\
\hline Población total según derechohabiencia a servicios de salud & $3,801,962$ & 100.00 \\
\hline Derechohabiente & $2,129,000$ & 56.00 \\
\hline No derechohabiente & $1,637,908$ & 43.08 \\
\hline No especificó & 35,054 & 0.92 \\
\hline Población en pobreza & $2,566,157$ & 67.40 \\
\hline Población en pobreza extrema & $1,135,230$ & 29.80 \\
\hline Municipios & 2010 & \\
\hline Santa María Colotepec & Valor absoluto & $\%$ \\
\hline Población total & 22,562 & - \\
\hline PEA & 8,250 & 100.00 \\
\hline Desocupados & 395 & 4.79 \\
\hline Ocupados & 7,855 & 100.00 \\
\hline PEA ocupada con ingresos de hasta dos salarios mínimos & 3,577 & 45.54 \\
\hline Población total según derechohabiencia a servicios de salud & 22,562 & 100.00 \\
\hline Derechohabiente & 15,082 & 66.85 \\
\hline No derechohabiente & 7,415 & 32.86 \\
\hline No especificó & 65 & 0.29 \\
\hline Población en pobreza & 18,651 & 75.60 \\
\hline Población en pobreza extrema & 7,125 & 28.90 \\
\hline Santa María Tonameca & Valor absoluto & $\%$ \\
\hline Población total & 24,318 & - \\
\hline PEA & 7,397 & 100.00 \\
\hline Desocupados y tasa de desocupación & 174 & 2.35 \\
\hline Ocupados & 7,223 & 100.00 \\
\hline PEA ocupada con ingresos de hasta dos salarios mínimos & 4,913 & 68.02 \\
\hline Población total según derechohabiencia a servicios de salud & 24,318 & 100.00 \\
\hline Derechohabiente & 15,188 & 62.46 \\
\hline No derechohabiente & 9,056 & 37.24 \\
\hline No especificó & 74 & 0.30 \\
\hline Población en pobreza & 18,925 & 87.30 \\
\hline Población en pobreza extrema & 12,267 & 56.60 \\
\hline
\end{tabular}

Fuente: elaboración con datos del Censo de Población y Vivienda 2010 del INEGI y del Coneval. 
Estos elevados niveles de marginación y pobreza se reflejan en los serios rezagos que presenta la infraestructura de los hogares tanto en Oaxaca como en los municipios considerados, pues se evidencian diferencias muy grandes que muestran las severas carencias que padecen los hogares oaxaqueños en relación con los datos nacionales (véase cuadro 3).

\section{CUADRO 3}

Características de la vivienda para Oaxaca y municipios seleccionados, 2010 (porcentajes)

\begin{tabular}{lcccc}
\hline $\begin{array}{c}\text { Nacional/entidad/ } \\
\text { municipio }\end{array}$ & $\begin{array}{c}\text { Con algún } \\
\text { nivel de } \\
\text { hacinamiento }\end{array}$ & $\begin{array}{c}\text { Sin agua } \\
\text { entubada }\end{array}$ & $\begin{array}{c}\text { Sin energía } \\
\text { eléctrica }\end{array}$ & $\begin{array}{c}\text { Sin drenaje ni } \\
\text { servicio sanitario } \\
\text { exclusivo }\end{array}$ \\
\hline Nacional & 36.53 & 8.63 & 1.77 & 3.57 \\
Oaxaca & 46.53 & 23.66 & 4.93 & 4.01 \\
$\quad$ Santa María Colotepec & 56.04 & 25.79 & 4.15 & 3.10 \\
Santa María Tonameca & 61.56 & 50.30 & 7.91 & 7.89 \\
\hline
\end{tabular}

Fuente: elaboración propia con datos de Conapo.

Bajo este contexto socioeconómico se ha detonado el fenómeno de la migración y su crecimiento acelerado en Oaxaca, tal como lo advierte Alvarado (2008: 88): «La falta de desarrollo económico y la confluencia de otros fenómenos generan el escenario propicio para que se dé el flujo migratorio».

La migración y remesas en cuatro comunidades de la costa oaxaqueña

En total se encuestaron 223 hogares en cuatro comunidades de la costa oaxaqueña, de las cuales dos pertenecen al municipio de Santa María Tonameca: Vainilla y Escobilla; y las restantes, Barra de Navidad y Ventanilla, pertenecen al municipio de Santa María Colotepec. En esas comunidades 43 por ciento de los hogares (95) cuenta con algún migrante; la comunidad donde existe una mayor participación de migrantes es Escobilla y la que presenta el menor nivel de hogares con familiares migrantes es Ventanilla (gráfica 1).

$128 \frac{\text { SEGUNDO SEMESTRE } 2016}{\text { MIGRACIÓN Y DESARROLLO NÚM. } 27}$ 
GRÁFICA 1

Proporción de los hogares con algún migrante en las comunidades consideradas (porcentajes)

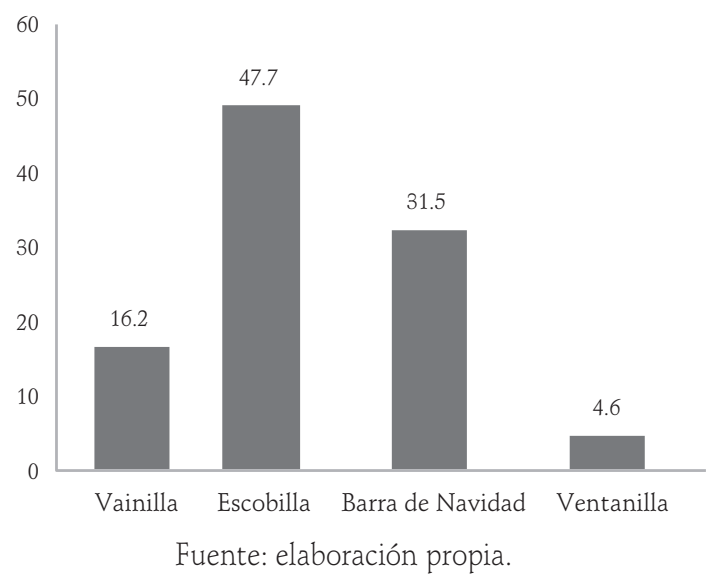

De los 95 hogares que tenían al menos un familiar migrante, en promedio habitan alrededor de cuatro personas por hogar, tienen una edad promedio de 34 años y los habitantes mayores de 15 años cuentan con cinco años de educación. De estos hogares se obtuvo información que indica la existencia de 130 migrantes, de los cuales 81 por ciento reside en Estados Unidos, mientras que 19 por ciento son migrantes internos.

Los principales destinos en Estados Unidos fueron Ohio y Texas, este último es reconocido como un destino tradicional de las migraciones de mexicanos (Passel y Suro, 2005), mientras que el estado de Ohio se ha convertido en un nuevo lugar de recepción de acuerdo con la investigación presentada por Cohen y Chávez (2013). Concerniente a la migración interna, los destinos primordiales fueron Pochutla, Oaxaca, Puerto Escondido y Distrito Federal, regiones que revelan una considerable dinámica económica y que son importante polos de atracción de la migración interna, debido a que las fuerzas de aglomeración tienen un impacto geográfico sobre la migración por la concentración de la actividad económica en unas pocas regiones (Crozet, 2004).

Las migraciones hacia Estados Unidos se caracterizan por ser de reciente incorporación al flujo de migración internacional, ya que partieron sobre todo en 
el periodo precrisis (2000-2006) y han mantenido un flujo de migración relevante incluso después de la crisis inmobiliaria de 2007 (gráfica 2).

\section{GRÁFICA 2}

Periodo en el cual se marchó por primera

vez su familiar migrante (porcentajes)

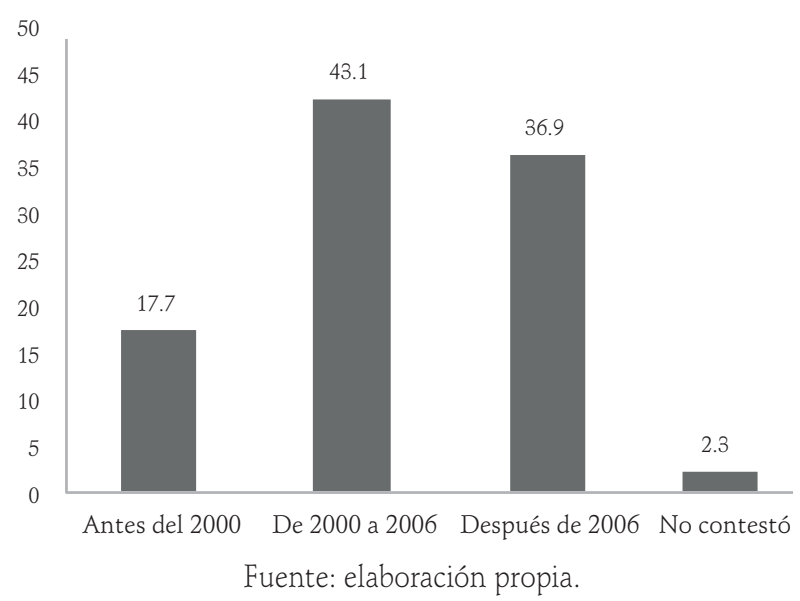

Se detectó que 73 por ciento de esos hogares que contaban con algún familiar migrante declararon recibir remesas durante 2013, el monto que señalaron percibir en promedio fue cercano a los 22 mil pesos anuales (alrededor de mil 800 pesos mensuales) y el uso principal que hicieron de este recurso fue para el gasto corriente del hogar (54.6 por ciento) (gráfica 3). 


\section{GRÁFICA 3}

Uso de las remesas (porcentajes)

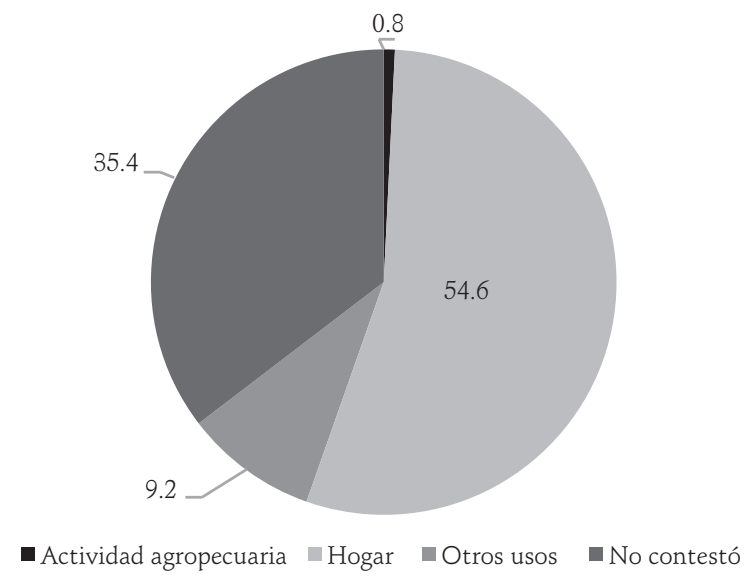

Fuente: Elaboración propia.

\section{Comparativo infraestructura del hogar}

Para llevar a cabo un análisis sobre el posible impacto que las remesas pueden tener sobre la infraestructura de los hogares receptores, tal como lo sugiere la visión funcionalista de las remesas; consideramos llevar a cabo un ejercicio estadístico para validar dicha teoría, con tal finalidad tomamos a los hogares que cuentan con un familiar migrante y los comparamos con los que no tenían un familiar migrante. Consideramos que manejar esta división entre los hogares nos permitirá obtener una mayor información respecto al impacto que tienen las remesas sobre la infraestructura y actividades productivas del hogar.

Al realizar un análisis comparativo sobre la infraestructura y acceso a servicios por parte de los dos tipos de hogares considerados, encontramos que no existían diferencias importantes, situación que es respaldada con una prueba de hipótesis sobre la comparación proporcional entre categorías de grupos (en este caso hogares con migrantes y sin migrantes) por medio del estadístico ji-cuadrado 
de Pearson. ${ }^{2}$ Los resultados obtenidos con relación a los materiales empleados en los techos de ambos grupos de hogares, el estadístico señaló que no existían diferencias significativas entre ambos grupos (a un nivel de significación estadística del 5 por ciento); situación que se refleja en el gráfico 4, pues los datos de los tipos de hogar tienen un comportamiento muy similar.

\section{GRÁFICA 4}

Materiales empleados en el techo del hogar (porcentajes)

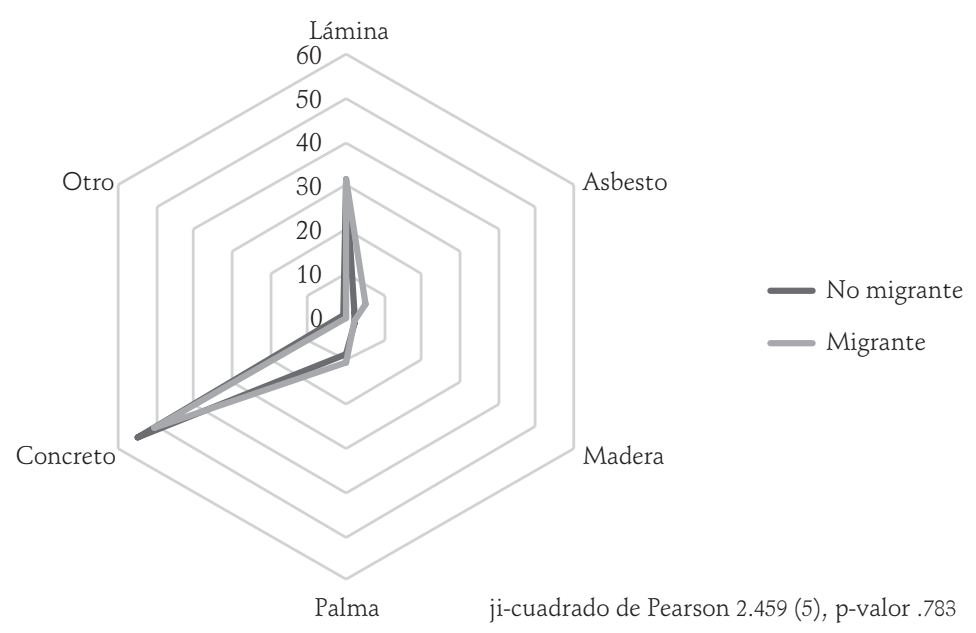

Fuente: elaboración propia.

En el caso de las viviendas, cerca de 92 por ciento de los hogares con un familiar migrante tenían paredes de concreto, respecto a 88 por ciento de los hogares que no contaban con migrantes (gráfica 5), diferencia que no fue significativa según estadístico ji-cuadrada de Pearson.

Referente al material empleado en los pisos, cerca de 90 por ciento de los hogares con un familiar migrante tenían pisos elaborados con materiales durables como el concreto, en comparación con 83 por ciento de los otros hogares

${ }^{2}$ El estadístico de ji-cuadrada para tablas de contingencia es una prueba no paramétrica para determinar si dos grupos distintos e independientes difieren en la frecuencia de una variable dependiente categórica (Landau y Everitt, 2004). 
(gráfica 6), diferencia que no fue significativa entre ambos grupos de hogares de acuerdo con el estadístico ji-cuadrado de Pearson.

\section{GRÁFICA 5}

Materiales empleados en las paredes del hogar (porcentajes)

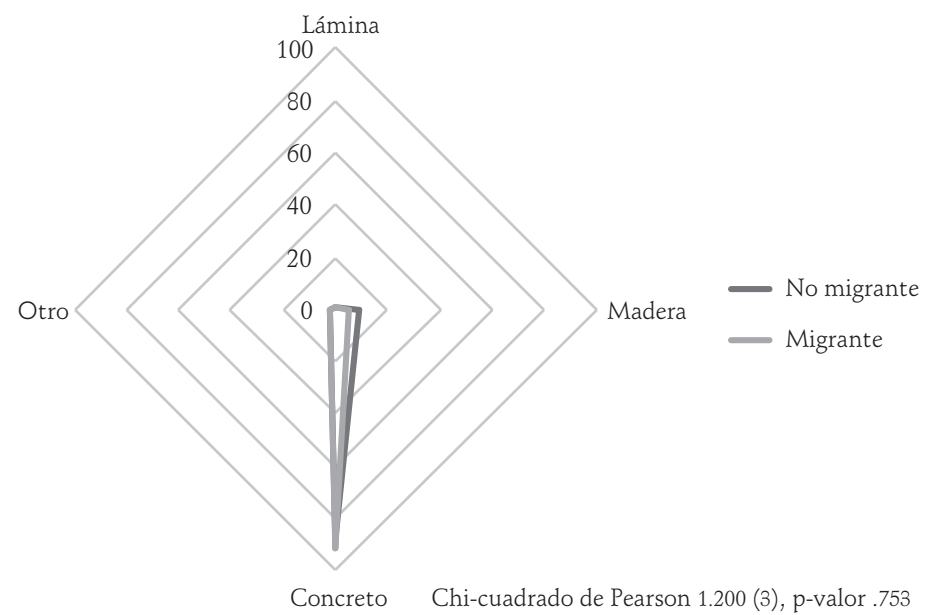

Fuente: elaboración propia.

\section{GRÁFICA 6}

Materiales empleados en los pisos del hogar (porcentajes)

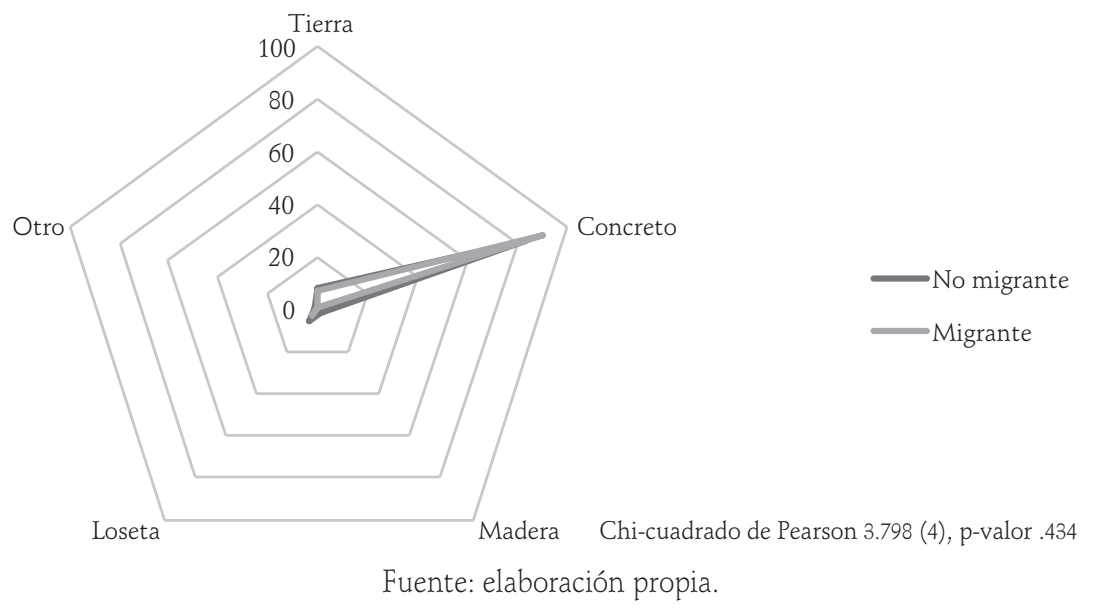


En cuanto al acceso a los servicios de drenaje en ambos tipos de hogares, la mayoría contaba con una fosa séptica, hoyo negro y letrina, con proporciones muy similares (gráfica 7), situación que se corrobora con el estadístico ji-cuadrada.

\section{GRÁFICA 7}

Acceso a los servicios de drenaje (porcentajes)

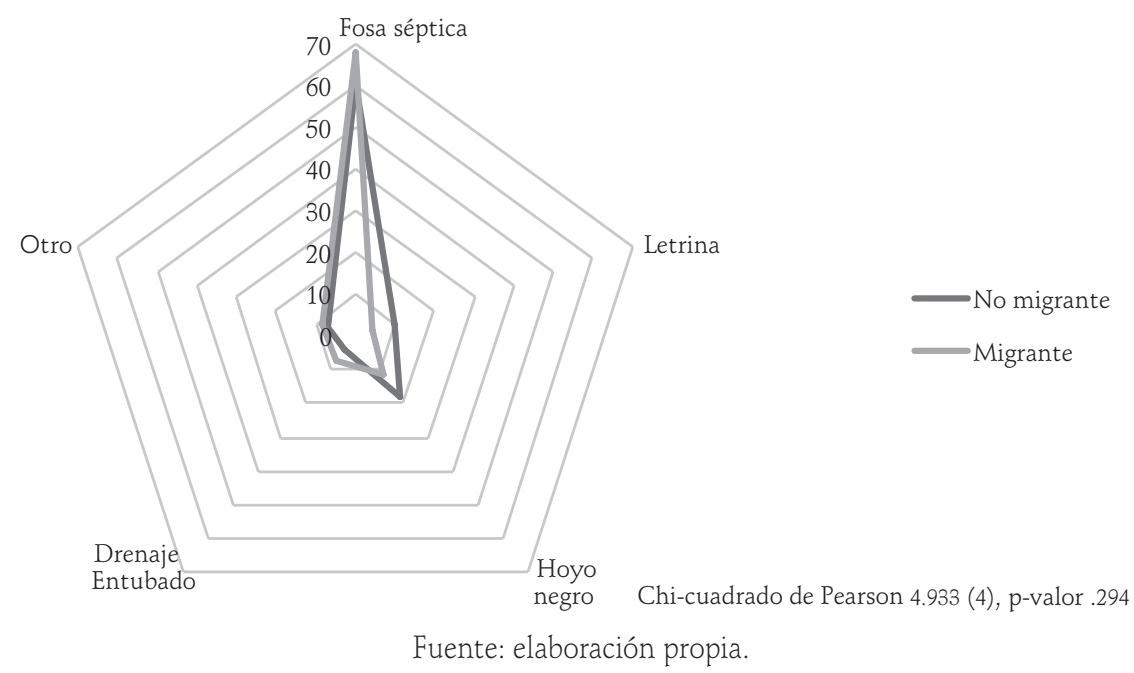

En torno a la infraestructura es posible comentar que no existen diferencias significativas entre ambos grupos de hogares; ahora bien, la visión funcionalista de las remesas indica que además de contribuir con la infraestructura de los hogares, dichos recursos pueden invertirse en forma productiva; sin embargo, en este trabajo nos interesa ampliar dicho concepto más allá de los límites de la visión funcionalista. En ese sentido, queremos analizar si dichos emprendimientos productivos son sustentables, de confirmarse esto, las remesas tendrían un impacto positivo sobre el desarrollo sustentable de las comunidades.

Al analizar las actividades productivas que se realizan en ambos tipos de hogares se consideraron dos categorías fundamentales: 1) hogar diversificado productivamente, es decir si el hogar realiza más de una actividad que le genere 
ingresos (se consideraron actividades agrícolas, ganaderas, comercio, pesca, etcétera; 2) hogar no diversificado productivamente, que comprende una sola actividad económica. A estas dos categorías se agregan las etiquetas de sustentable, si dentro de esas actividades reportaron llevar a cabo la práctica del ecoturismo o bien no sustentable. Así que la combinación de esas categorías brinda cuatro posibles categorías para ambos tipos de hogares (gráfica 8). Cabe resaltar que de acuerdo con el estadístico ji-cuadrado de Pearson no se encontró evidencia de una diferencia significativa a 5 por ciento en torno al tipo de actividades productivas entre ambos tipos de hogares; en otras palabras, los hogares que reciben remesas no tienden a mostrar una mayor diversificación productiva hacia prácticas sustentables como el ecoturismo con relación a los hogares no receptores, por tal motivo la contribución de las remesas sobre el desarrollo sustentable es prácticamente nula, pero tampoco es una limitante. En efecto: el ser un hogar con migrantes no limita la posibilidad de hacer ecoturismo. Aunado a lo anterior, todos los hogares están diversificados, lo cual quiere decir que la migración tampoco determina el tener mayor o menor diversidad de actividades productivas. Esto es diferente a otros estudios que han indicado la importancia de la migración en los medios de vida de los hogares rurales (Berhaus et al., 2007; Mushongah y Scoones, 2012). Las estrategias esenciales de los hogares rurales señaladas en la literatura son la migración, la diversificación y la intensificación (Ellis, 2000, citado en Mushongah y Scoones, 2012), y es interesante notar en los resultados que tales estrategias no son excluyentes unas de otras.

Los datos muestran que el impacto de las remesas sobre el desarrollo, sustentable o no, es prácticamente nulo, lo que podría explicarse por el hecho de que la mayor parte de los recursos se destina al consumo de los hogares, tal como lo advierte la visión estructuralista (Binford 2003; Aragonés y Salgado, 2011b). 


\section{GRÁFICA 8}

Diversificación productiva de las remesas en ambos tipos de hogares (porcentajes)

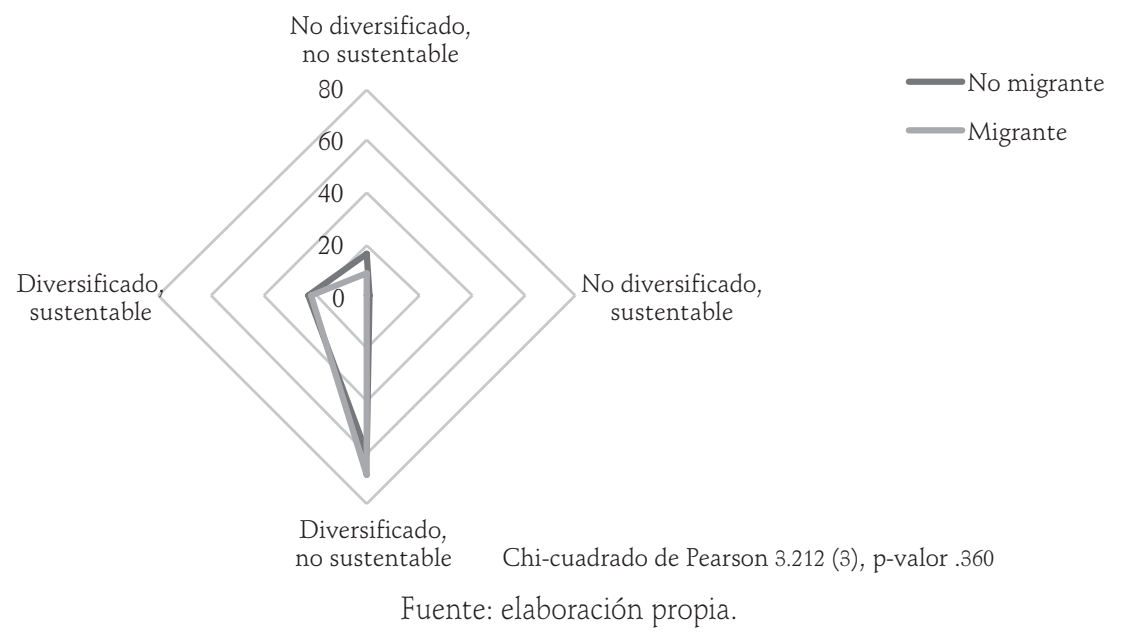

Sin embargo, la literatura reciente acerca de las remesas expone que si bien los flujos monetarios no han logrado detonar mayores niveles de calidad de vida en las regiones receptoras, pueden tener impactos a nivel de cambios en los patrones de consumo entre los dos tipos de hogares (Davis y López-Carr, 2010). Dicha situación genera efectos posteriores en forma indirecta, con el uso de los recursos naturales en las comunidades receptoras (Oin y Flint, 2012). Esta nueva perspectiva nos permitió efectuar un análisis para averiguar si existen evidencias significativas en torno a los patrones de consumo en ambos hogares.

Patrones de consumo entre los hogares

Se informa que el ingreso que perciben los hogares receptores de remesas tiende a provocar modificaciones en los patrones de consumo de los hogares por medio de lo que se conoce como «remesas sociales», las cuales se relacionan con ideas, comportamientos, identidades y capital social que fluye desde los lugares

$136 \frac{\text { SEGUNDO SEMESTRE } 2016}{\text { MIGRACIÓN Y DESARROLLO NÚM. } 27}$ 
receptores de migrantes hacia las regiones expulsoras (Levitt, 1998). Este tipo de recurso puede influir y dar forma al consumo conspicuo, que se define como la compra de bienes y servicios con el propósito específico de mostrar la riqueza personal o el estatus social, en especial cuando los bienes y servicios adquiridos son demasiado caros para los otros miembros de las diversas clases sociales (Veblen, 2007).

Ahora bien, esos cambios en el patrón de consumo podrían repercutir en el uso de los recursos naturales en el entorno de la comunidad. Por un lado, el incremento en el nivel de ingresos en los hogares receptores puede tener un impacto positivo sobre el medio ambiente, debido a que permitirá sustituir los bienes locales por otro tipo de bienes o recursos fuera de su ecosistema (fuentes de energía distintas a las maderas como combustibles) contribuyendo de manera positiva en su biodiversidad (Qin y Flint, 2012). Sin embargo, las remesas también pueden tener impactos ambientales negativos, cuando se estimula el consumo de recursos naturales, lo que provoca mayores tasas de depredación. $O$ bien se invierte en prácticas no sustentables con el medio ambiente, tal como el uso de fertilizantes químicos y la conversión de bosques al pastoreo u otras actividades comerciales no sustentables (Adger et al., 2002).

En ese sentido, existen pocos estudios que aborden esta línea de investigación para el caso mexicano, uno de ellos es el presentado por Klooster (2003), quien expresa que los hogares receptores de remesas en Michoacán las emplean para comprar gas propano importado como combustible, reduciendo así el consumo de los recursos forestales cercanos a la comunidad como fuente primaria de energía, ello ha permitido llevar a cabo una conservación forestal en la región.

En gran parte de la literatura relativa al estudio de las remesas y sus posibles usos se aprecia que el consumo corriente del hogar es el principal destino de los gastos, desde los trabajos de Jorge Durand (1996) que explicaban que en diversas comunidades del país alrededor de 76 por ciento de los dólares recibidos se destinaban a ese rubro; un resultado similar aparece en el trabajo de Zarate-Hoyos (2004), en el que se exhibe que los hogares rurales de diversas regiones de México invertían casi 76 por ciento de las remesas en el gasto diario de los hogares receptores; para el caso de Oaxaca, el trabajo de Cohen y 
Rodriguez (2005) refiere que aproximadamente 92 por ciento de los hogares receptores de remesas empleaban ese recurso para cubrir los costos de la vida diaria. En nuestro trabajo encontramos resultados similares para el caso de las comunidades ubicadas en la costa oaxaqueña.

Por tanto, en esta sección extendimos el análisis hacia la diferenciación de los patrones de consumo entre los dos tipos de hogares considerados en el estudio y encontramos algunas diferencias significativas que se sustentan con las pruebas de hipótesis construidas a partir del estadístico ji-cuadrado de Pearson.

En el siguiente gráfico se observa que el consumo de bienes y servicios considerados como conspicuos se presentan varias diferencias interesantes, correspondientes a los servicios de luz eléctrica, adquirida por 99 por ciento de los hogares con un familiar migrante, respecto a 93 por ciento de los que no tienen familiar migrante; la prueba de hipótesis indica que existe una diferencia relevante entre los dos grupos de hogares (véase anexo estadístico A). De igual modo, la adquisición de telefonía fija tiene una mayor participación en los hogares que cuentan con un familiar migrante, cerca de 38 por ciento tenía ese servicio, con referencia a 14 por ciento de los hogares sin familiar migrante, que se valida según el estadístico ji-cuadrado de Pearson (anexo estadístico A). El tercero y último rubro comprende el uso de gas para cocinar: 71 por ciento de los hogares que cuentan con un familiar migrante lo utilizan, en contraste con 57 por ciento de los hogares sin familiar migrante que lo ocupan; la prueba de hipótesis revela que la diferencia es significativa (anexo estadístico A).

Es posible esperar que este tipo de consumo cause impactos en el uso de los recursos naturales, pues la mayor participación de los hogares con un familiar migrante en cuanto al uso de gas como combustible para cocinar podría contribuir con la biodiversidad de los bosques en las regiones estudiadas. Esto lo resaltamos como una propuesta de investigación a futuro en la región costera de Oaxaca porque el alcance de nuestra investigación no permite corroborar si en efecto se ha evidenciado recuperación de bosque, como en el estudio de caso en Michoacán presentado por Klooster (2003) o el de San José La Arada en Guatemala, desarrollado por Holder y Chase (2011). 


\section{GRÁFICA 9}

Acceso a aparatos electrodomésticos y servicios

de telecomunicaciones (porcentajes)

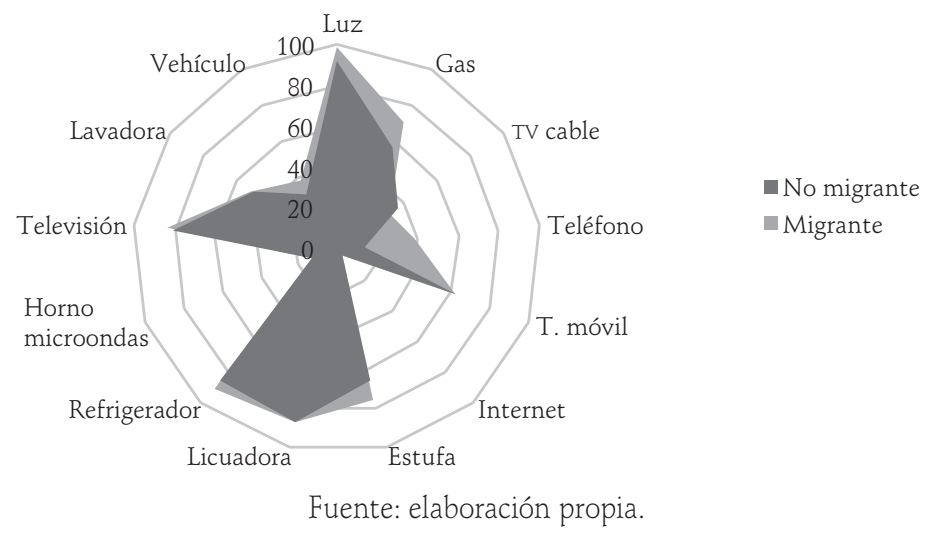

\section{Conclusiones}

Gran parte de la literatura que se centra en examinar los impactos de las remesas en las comunidades de origen se han enfocado en estudiar exclusivamente la relación migración y desarrollo, dejando fuera del análisis las probables implicaciones que dicho recurso puede tener sobre el medio ambiente. Debido a esa situación, de modo reciente han surgido investigaciones que amplían la discusión hacia el uso de los recursos naturales en las comunidades de origen, de ahí la trascendencia de estudiar el papel que desempeñan en el contexto del desarrollo sustentable.

Los resultados del análisis comparativo por tipo de hogar muestran que la capacidad de las remesas para influir en los niveles de desarrollo, sea sustentable o no, son muy limitadas; la mayor proporción del recurso se gasta en el consumo corriente del hogary es muy poca la proporción invertida en emprendimientos productivos. Adicionalmente, cabe destacar que no se observan diferencias significativas entre los mismos grupos de hogares en torno a la diversificación sustentable, lo que indica que no se promueve, pero tampoco la limita. Incluso la migración y la diversificación son estrategias de vida complementarias de los 
hogares rurales, por lo que el desplazamiento de población se considera una estrategia de diversificación de los ingresos en el hogar.

A su vez, los resultados del estudio de caso para la costa oaxaqueña indican que las remesas han provocado un cambio en el patrón de consumo en los hogares receptores, cuyas consecuencias son directas sobre el uso de los recursos naturales cercanos a la comunidad, ello sugiere que puede existir un efecto positivo en el medio ambiente de dichas comunidades receptoras; no obstante, esto poco o nada tiene que ver con el desarrollo sustentable: mientras el dinero fluya hacia esos hogares podrán sustituir el uso de los recursos naturales locales por el de otras regiones, pero si el flujo disminuye o se interrumpe volverán a recurrir a los recursos locales.

\section{Bibliografía}

ADELMAN, Irma y Edward Taylor (1990), "Is structural adjustment with a human face possible? The case of Mexico", The Journal of Development Studies, vol 26, no. 3.

ADGER, Neil, Mick Kelly, Alexandra Winkels, Quang Huy y Catherine Locke (2002), "Migration, remittances, livelihood trajectories, and social resilience», AMBIO: A Journal of the Human Environment, vol 31, no. 4.

Alvarado, Ana María (2008), "Migración y pobreza en Oaxaca», El Cotidiano, Universidad Autónoma Metropolitana-Unidad Azcapotzalco, vol. 23, núm. 148.

ARAGONÉS, Ana María (2009), "El trabajo-exportador y las inversiones extranjeras», Comercio Exterior, vol. 59, núm. 1.

ARAGONÉS, Ana María y Uberto Salgado (2011a), Nuevo patrón migratorio bajo el contexto de la crisis, en Ana María Aragonés (coord.), "Crisis económica y migración. i̇Impactos temporales o estructurales?, México, Universidad Nacional Autónoma de México.

ARAGONÉS, Ana María y Uberto Salgado (2011b), «żPuede la migración ser un factor para el desarrollo de los países expulsores?», Migración y desarrollo, vol. 9, núm. 17.

ARAGOnÉS, Ana María, Uberto Salgado y Esperanza Ríos (2008), «żA quién benefician las remesas?’, Economía, Universidad Nacional Autónoma de México, vol. 5, núm. 14. 
Berhaus, Wassie, David Colman y B. Bichaka Fayissa (2007), «Diversification and livelihood sustainability in a Semi-arid Environment: a case study from southern Ethiopia", Journal of Development Studies, vol. 43, no. 5.

BILSBORROW, Richard (2015), "Cambio climático y migración», en Ana María Aragonés (coord.), Consecuencias del cambio climático sobre la migración. Un análisis para México, México, Universidad Nacional Autónoma de México.

Binford, Leigh (2002), "Remesas y subdesarrollo en México», Relaciones. Estudios de historia y sociedad, Michoacán, México, vol. XXIII, núm. 90. (2003), «Migrant remittances and (under) development in Mexico», Critique of Anthropology, vol. 23, no. 3 .

CARABIAS, Julia, José Sarukhán, Javier de la Maza y Carlos Galindo (2010), Patrimonio natural de México. Cien casos de éxito, México, Comisión Nacional para el Conocimiento y uso de la biodiversidad.

CERÓN, Hazael y Antonio Yúnez-Naude (2015), «Diversificación en la economía rural hacia actividades no agropecuarias y sus impactos en pobreza y desigualdad», en Antonio Yúnez-Naude, Fabiola Rivera, María de los Ángeles Chávez, Jorge Mora y Edward Taylor (coords.), La economía del campo mexicano: tendencias y retos para su desarrollo, México, El Colegio de México.

COHEN, Jeffrey y Leila Rodriguez (2005), «Remittance outcomes in rural Oaxaca, Mexico: Challenges, options and opportunities for migrant households», Population, Space and Place, vol. 11, no. 1.

CoHeN, Jeffrey y Merino Chavez (2013), "Latino immigrants, discrimination and reception in Columbus, Ohio», International Migration, vol. 51, no. 2.

Comité Estatal de Información Estadística y Geográfica (CEIEG) (2014), Boletín económico Oaxaca, Oaxaca, México, Comité Estatal de Información Estadística y Geografía.

Consejo Nacional de Población (2014), Índice Absoluto de Intensidad Migratoria México Estados Unidos. 2000-2010, México, Conapo.

CROZET, Matthieu (2004), "Do migrants follow market potentials? An estimation of a new economic geography model», Journal of Economic Geography, vol. 4, no. 4.

DAVIS, Jason y David Lopez-Carr (2010), «The effects of migrant remittances on population-environment dynamics in migrant origin areas: international migra- 
tion, fertility, and consumption in highland Guatemala», Population and Environment, vol. 32, no. 2-3.

DuRAND, Jorge, Emilio Parrado y Douglas Massey (1996), «Migradollars and development: a reconsideration of the Mexican case», International Migration Review, vol. XXX.

Fierros, Isael y Sophie Avila-Foucat (en revisión), «Livelihood profiles, assets and vulnerability contexts in Mexican Rural Households».

FOLADORI, Guillermo (1999), Los limites del desarrollo sustentable, Montevideo, Ediciones de la Banda Oriental.

FOLADORI, Guillermo y Humberto Tommasino (2000), «El concepto de desarrollo sustentable treinta años después», Desenvolvimento e Meio Ambiente, núm. 1.

FOSTER, Bellamy (1994), The vulnerable planet: A short economic history of the environment, NYU Press.

GHAI, Dharam (2003), «Trabajo decente. Concepto e indicadores», Migración internacional del trabajo, vol. 122, núm. 2.

Hecht, Susanna, Susan Kandel y Abelardo Morales (2012), Migración, medios de vida rurales y manejo de recursos naturales, Programa Salvadoreño de Investigación sobre Desarrollo y Medio Ambiente, Centro Internacional de Investigaciones para el Desarrollo de Canadá y la Fundación Ford, El Salvador.

HOLDER, Curtis y Gregory Chase (2012), «The role of remittances and decentralization of forest management in the sustainability of a municipal-communal pine forest in eastern Guatemala», Environment, development and sustainability, vol. 14, no. 1.

Instituto Nacional de Estadística y Geografía (INEGI) (2016), «PIB-Entidad Federativa anual», en http://www.inegi.org.mx/est/contenidos/proyectos/cn/pibe/ (12/05/2016).

JONES, Richard (1995), Ambivalent journey: US migration and economic mobility in NorthCentral Mexico, Arizona, University of Arizona Press.

Klooster, Dan (2003), «Forest transitions in Mexico: institutions and forests in a globalized countryside», The Professional Geographer, vol. 55, no. 2.

LANDAU, Sabine y Brian Everitt (2004), A handbook of statistical analyses using SPSS, Boca Raton, FL, Chapman \& Hall/CRC.

LEVITT, Peggy (1998), «Social remittances: Migration driven local-level forms of cultural diffusion», International Migration Review. 
LiPTON, Michael (1980), «Migration from rural areas of poor countries: the impact on rural productivity and income distribution», World Development, vol. 8, no. 1.

LóPEZ-FELDMAN, Alejandro (2014), "Shocks, income and wealth: do they efect the extraction of natural resources by rural households?, World Development, vol. 64, no. S1.

MASSEY, Douglas y Emilio Parrado (1998), «International migration and business formation in Mexico", Social Science Quarterly.

MUSHONGAH, Josphat e Ian Scoones, (2012), "Livelihood change in rural Zimbabwe over 20 years", Journal of Development Studies, vol. 48, no. 9.

NAVA EsCudero, César (2013), Ciencia, ambiente y derecho, México, Universidad Nacional Autónoma de México.

Organización de las Naciones Unidas (ONU) (2015), «Objetivos de desarrollo sustentable», en http://www.un.org/sustainabledevelopment/es/objetivos-de-desarrollosostenible/ (12/05/2016).

PASSEL, Jeffrey y Roberto Suro (2005), Rise, peak, and decline: Trends in US immigration 1992-2004, Washington, DC, Pew Hispanic Center.

OIN, Hua y Courtney Flint (2012), «Integrating rural livelihoods and community interaction into migration and environment research: a conceptual framework of rural out-migration and the environment in developing countries», Society \& $\mathrm{Na}$ tural Resources, vol. 25, no. 10.

SHERBInin, Alex et al. (2008), "Rural Household Demographics, Livelihoods and the Environment», author manuscript, en http://www.ncbi.nlm.nih.gov/pmc/articles/PMC2351958/ (12/05/2016).

Simmons, Ian (1993), Environmental history: A concise introduction, Oxford, Blackwell.

STEFoni, Carolina (2011), «Migración, remesas y desarrollo. Estado del arte de la discusión y perspectivas», Polis (Santiago), vol. 10, núm. 30.

STIGLITZ, Joseph (2006), ¿Cómo hacer que funcione la globalización?, México, Santillana.

VEBLEN, Thorstein (2007), The theory of the leisure class, OUp Oxford.

World Commission on Environment and Development (WCED) (1987), Our common future, Oxford, Oxford University Press.

ZARATE HOYOS, German (2004), "Consumption and remittances in migrant households: toward a productive use of remittances», Contemporary Economic Policy, vol. 22, núm. 4. 


\section{Anexo estadístico A}

\section{CUADRO 4}

\begin{tabular}{lccc}
\hline \multicolumn{1}{c}{ Tipos de bienes } & $\begin{array}{c}\text { Chi-cuadrado de } \\
\text { Pearson }\end{array}$ & gl & p-valor \\
\hline Vehículo & 1.16 & 1 & 0.282 \\
Lavadora & 0.079 & 1 & 0.778 \\
Televisión & 0.252 & 1 & 0.616 \\
Horno microondas & 1.200 & 1 & 0.273 \\
Refrigerador & 1.024 & 1 & 0.312 \\
Licuadora & 0.006 & 1 & 0.939 \\
Estufa & 2.087 & 1 & 0.149 \\
\hline Tipos de servicios & Chi-cuadrado de & gl & p-valor \\
\hline Luz & Pearson & 1 & 0.034 \\
Gas & 4.474 & 1 & 0.046 \\
TV de cable & 3.965 & 1 & 0.540 \\
Telefóno & 0.376 & 1 & 0.000 \\
Teléfono móvil & 15.783 & 1 & 0.914 \\
Internet & 0.012 & 1 & 0.645 \\
\hline
\end{tabular}

Fuente: elaboración propia. 